\title{
The Contrastive Research in the Photocatalytic Activity of BiOBr Synthesized by Different Reactants
}

\author{
Wang Ling-Li,, ${ }^{1}$ Ma Wan-Hong, ${ }^{1}$ Wang Shu-Lian, ${ }^{1}$ Zhang Yu, ${ }^{1}$ Jia Man-Ke, ${ }^{1}$ Li Rui-Ping, \\ Zhang Ai-Qing, ${ }^{2}$ and Huang Ying-Ping ${ }^{1}$ \\ ${ }^{1}$ Engineering Research Center of Eco-Environment in Three Gorges Reservoir Region, China Three Gorges University, \\ Ministry of Education, Yichang 443002, China \\ ${ }^{2}$ Key Laboratory Catalysis and Materials Science of the State Ethnic Affairs Commission, \\ China South-Central University for Nationalities, Ministry of Education, Wuhan 430071, China
}

Correspondence should be addressed to Huang Ying-Ping, chem_ctgu@126.com

Received 17 July 2012; Accepted 3 August 2012

Academic Editor: Chunyi Zhi

Copyright (๑) 2012 Wang Ling-Li et al. This is an open access article distributed under the Creative Commons Attribution License, which permits unrestricted use, distribution, and reproduction in any medium, provided the original work is properly cited.

$\mathrm{BiOBr}$ nanoplates, marked as $\alpha-\mathrm{BiOBr}$ and $\beta$ - $\mathrm{BiOBr}$, were synthesized via hydrothermal method using cetylpyridinium bromide $(\mathrm{CPB})$ and $\mathrm{NaBr}$ as reactants, respectively. X-Ray Diffraction (XRD), transmission electron microscope (TEM), $\mathrm{N}^{2}$ adsorption/desorption, UV-visible diffuse reflectance spectroscopy (UV-Vis DRS), and cyclic voltammetry (CV) were employed to characterize the obtained $\mathrm{BiOBr}$. The results showed that $\alpha$ - $\mathrm{BiOBr}$ and $\beta$ - $\mathrm{BiOBr}$ can absorb visible light and both the band gaps of them were about $2.76 \mathrm{eV}$. Under visible light irradiation, the photodegradation of organic dye sulforhodamine (SRB) and salicylic acid (SA) using $\alpha-\mathrm{BiOBr}$ and $\beta$ - $\mathrm{BiOBr}$ as the catalysts was carried out. The reaction kinetic constants of the degradation of SRB by $\alpha-\mathrm{BiOBr}$ and $\beta$-BiOBr were $0.00602 \mathrm{~min}^{-1}$ and $0.0047 \mathrm{~min}^{-1}$, respectively, which indicated that the photocatalytic activity of $\alpha-\mathrm{BiOBr}$ was higher than that of $\beta$-BiOBr. The UV-Vis DRS and total organic carbon (TOC) were also monitored, and the TOC removal rate of $\alpha-\mathrm{BiOBr}$ and $\beta-\mathrm{BiOBr}$ was $86 \%$ and $48 \%$, respectively. At the same time, hydrogen peroxide $\left(\mathrm{H}_{2} \mathrm{O}_{2}\right)$ and active radicals were measured and analyzed, which showed that the main active species was ${ }^{\circ} \mathrm{OH}$ during the photocatalytic reaction.

\section{Introduction}

The bismuth oxyhalides $(\mathrm{BiO} X, X=\mathrm{F}, \mathrm{Cl}, \mathrm{Br}, \mathrm{I})$ are a new family of semiconductor materials which have unique electronic structures, good optical properties, and catalytic performances, thus attract the interest of researchers $[1,2]$. Among them, the band gap of $\mathrm{BiOBr}$ [3-5] belongs to the visible region, so it is able to effectively use sunlight to degrade pollutants and overcome the shortage of the first-generation catalyst represented by $\mathrm{TiO}_{2}$ such as a low efficiency of solar energy and the strictly limited practical applications [6-8]. $\mathrm{BiOBr}$ is a semiconductor with highly anisotropic layered structure, and its electronic structure and band structure have been calculated through the DFT (density functional theory) method [9] and the TB-LMTO (tight-binding linear muffin-tin orbital) method [10]. The results indicated that $\mathrm{BiOBr}$ was the indirect transition band gap semiconductor, which in favor of hole-electron effective separation and charge transfer, so that $\mathrm{BiOBr}$ revealed a high photocatalytic activity.

The photocatalytic activity of semiconductor has a strong dependence on its particle size and morphology, so the synthesis of nanoparticles with a small size and shape is significant. Nowadays, the common methods to prepare nano$\mathrm{BiOBr}$ include hydrolysis method [11], soft-template method $[12,13]$, hydrothermal method $[14,15]$, and high-temperature solid-state method [16]. Introducing templates in preparation of catalysts is an effective technology to control the size and morphology. Templates provide a microreactor to control the prepared materials with same size and single scattered pore structure. So selecting a suitable template to synthesis $\mathrm{BiOBr}$ is an effective method to improve its catalytic activity. Cetyltrimethyl ammonium bromide (CTAB) [17, 18 ] is the most universal templates introduced in synthesis systems because it can be regarded as bromine sources and template simultaneously. But CPB which has the semblable 
effect with CTAB is seldom used to synthesis BiOBr. Herein, $\mathrm{CPB}$ is served as template and bromine sources to prepare $\mathrm{BiOBr}$ which compared with $\mathrm{BiOBr}$ synthesized using $\mathrm{NaBr}$ as bromine source, and the products were denoted as $\alpha$ $\mathrm{BiOBr}$ and $\beta$-BiOBr. The physical properties of products were characterized, and the photocatalytic activities of the two products were contrasted via degrading sulforhodamine (SRB) and salicylic acid (SA) under visible light irradiation. In the end, the photocatalytic mechanism of $\mathrm{BiOBr}$ photocatalysis was inferred via analyzing the active species during the catalytic process.

\section{Experimental}

2.1. Reagents and Instruments. Bismuth nitride $\left(\mathrm{Bi}\left(\mathrm{NO}_{3}\right)_{3}\right.$. $\left.5 \mathrm{H}_{2} \mathrm{O}\right) \mathrm{NaBr}$ and $\mathrm{CPB}$ were of A.R. grade from the Beijing Chemical Factory (China). The dye sulforhodamine-B was of spec-pure grade (Across). Horseradish peroxidase (POD) was purchased from Lizhudongfeng Biologic Technique, and N, N-diethylphenylenediamine (DPD) reagent was purchased from Merck. Other chemicals were of reagent grade and used without further purification. Deionized and doubly distilled water was used throughout the investigation. The following were also used.

JEM-2010 transmission electron microscopy (TEM) (Japan, JEOL); N/C 2100 TOC analyzer (Germany, Jena); F4500 fluorescence spectrometer (Japan, Hitachi); Lambda25 UV-visible spectrophotometer (U.S., PE); PHs-P pH meter (Mettler-Toledo Instruments Co., Ltd.); U-3010 UV spectrophotometer (Japan, Hitachi); 360 FTIR spectrometer (USA, Nicolet); ASAP 2020 model BET surface area and pore size analyzer (USA, Micrometritics); D8ADVANCE X-ray diffraction (Germany); ESR 300E spectrometer (Germany, Bruker); High performance liquid chromatograph (USA, Waters); AUTOLAB Electrochemical workstation (China, Chenhua).

2.2. Preparation of Nano-BiOBr. $\mathrm{Bi}\left(\mathrm{NO}_{3}\right)_{3} \cdot 5 \mathrm{H}_{2} \mathrm{O}$ solution was added dropwise to CPB solution $\left(n_{\mathrm{Bi}}: n_{\mathrm{Br}}=2: 3\right)$. The mix solution was stirred for $60 \mathrm{~min}$ and then transferred to a Teflon-lined autoclave to react for $17 \mathrm{~h}$ at $170^{\circ} \mathrm{C}$. When it was cooled to the room temperature, the product was centrifuged and washed with water and ethanol for many times, respectively, dried in a vacuum box for $5 \mathrm{~h}$, thus the photocatalyst was obtained and denoted as $\alpha-\mathrm{BiOBr}$.

As the above method, only CPB was converted into the same amount of $\mathrm{NaBr}$, and other conditions did not be changed. The obtained product was recorded as $\beta$-BiOBr.

2.3. Characterization. X-ray diffraction (XRD) patterns were conducted on a DMAX 2500 diffractometer equipped with a $\mathrm{Cu} K \alpha$ radiation at a scanning speed of $5 \% / \mathrm{min}$ ranging from 5 to $80^{\circ}$. Transmission electron microscopy (TEM) patterns were performed on a JEOL JEM-2010 electron microscope with a field emission gun operating at $200 \mathrm{kV}$. $\mathrm{N}_{2}$ adsorption-desorption isotherms were performed with a Quanta chrome Autosorb1-C system at $196^{\circ} \mathrm{C}$; the data were analyzed by the BET method. The UV-Vis spectroscopy was recorded at room temperature on a Hitachi UV-3010 spectrophotometer from 200 to $800 \mathrm{~nm}$. Cyclic voltammetry (CV) was carried out using Pt wire as the counter electrode, calomel electrode as the reference electrode, and glassy carbon electrode daubed $\mathrm{BiOBr}$ thin film as the working electrode. The electrolyte used was a solution of $0.2 \mathrm{~mol} / \mathrm{L}$ $\mathrm{Na}_{2} \mathrm{SO}_{4}$. The measurements were conducted on AUTOLAB Electrochemical Workstation. The potential scan range was from $-1.0 \mathrm{~V}$ to $+1.0 \mathrm{~V}$. The scan rate was $50 \mathrm{mv} / \mathrm{s}$ and scan cycle was 4 times.

2.4. Photocatalytic Processes and Analytical Methods. The degradation of organic dye SRB was utilized to characterize the photocatalytic activity of the various photocatalysts. $50 \mathrm{~mL}$ of $1.5 \times 10^{-5} \mathrm{~mol} / \mathrm{L} \mathrm{SRB}$ aqueous solution containing $10 \mathrm{mg} \mathrm{BiOBr}$ was added in $70 \mathrm{~mL}$ cylindrical rigid glass. The $\mathrm{pH}$ was adjusted to 3 with $\mathrm{HClO}_{4}$ solution $(1.0 \mathrm{~mol} / \mathrm{L})$. Prior to irradiation, the suspension was stirred for $1 \mathrm{~h}$ to achieve adsorption/desorption equilibrium. The system was irradiated under visible light $(\lambda>420 \mathrm{~nm})$. And the solution was sampled at diverse intervals. The liquid was centrifuged and the absorbance of the liquid was measured on a spectrophotometer (maximum absorbance at $565 \mathrm{~nm}$ ). The $A / A_{0} \sim t$ curve was plotted to the wavelength, where $A_{0}$, $A$ were absorbance of SRB at the initial and at reaction time $t$, respectively.

Determination of $\mathrm{H}_{2} \mathrm{O}_{2}$ [19]: $30 \mathrm{uL}$ of $0.1 \%$ POD solution, $1 \mathrm{~mL}$ of $\mathrm{KH}_{2} \mathrm{PO}_{4}-\mathrm{NaOH}$ buffer solution ( $\mathrm{pH} 6.8$ ), $150 \mathrm{uL}$ of $1.0 \%$ DPD solution, and $1 \mathrm{~mL}$ of reaction solution collected at different time intervals were added successively to $10 \mathrm{~mL}$ colorimetric tubes. The volume was constant at $5 \mathrm{~mL}$. After centrifugation, the absorbance was determined at $510 \mathrm{~nm}$.

The oxidative active radicals produced in the process of reaction were measured with the ESR method [20]. DMPO was used to capture paramagnetic species, and the ESR signals were recorded at room temperature. In order to reduce the experimental error, the same root quartz capillary was used during the whole process of measuring ESR. The measured parameters of free radicals were gained by direct measuring magnetic field and microwave frequency.

The TOC changes in different photocatalytic response time were measured with the N/C 2100TOC.

The infrared spectroscopy (IR) of photocatalytic reaction solution for $0 \mathrm{~h}, 1 \mathrm{~h}, 4 \mathrm{~h}, 8 \mathrm{~h}$ was evaporated in rotary evaporator with potassium bromide at $60^{\circ} \mathrm{C}$. Then the samples were dried and measured on an infrared spectrometer.

Photocatalytic degradation of SA: in $70 \mathrm{~mL}$ glass cylindrical hard, $25 \mathrm{~mL}$ SA that contained $20 \mathrm{mg}$ BiOBr was placed in dark $2 \mathrm{~h}$ to achieve the adsorption/desorption equilibrium. Then the solution was illuminated in visible light $(\lambda>$ $420 \mathrm{~nm}$ ) and carried out at different intervals. The concentration changes of samples were analyzed by high performance liquid chromatography analysis with a Waters 2998 photodiode array (PDA) detector and a $\mathrm{C}_{18}$ reverse-phase column (4.6 mm i.d. $\times 150 \mathrm{~mm}$, kromasil). The mobile phase was methanol and phosphate $(0.01 \mathrm{moL} / \mathrm{L}, \mathrm{pH} 3.5)$, and the ratio was $45: 55$. The inject volume of the sample was $20 \mu \mathrm{L}$ and the flow rate was $0.70 \mathrm{~mL} / \mathrm{min}$. The detection wavelength was $278 \mathrm{~nm}$. 


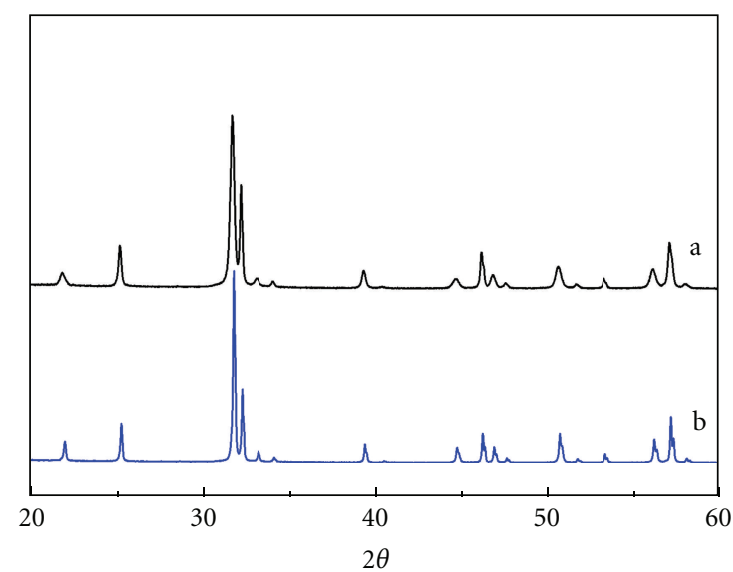

Figure 1: X-Ray diffraction pattern of BiOBr. (a) $\beta$-BiOBr; (b) $\alpha$-BiOBr.

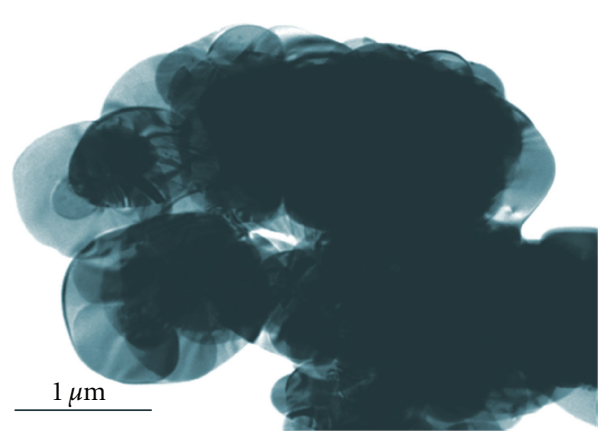

(a) $\alpha$ - $\mathrm{BiOBr}$

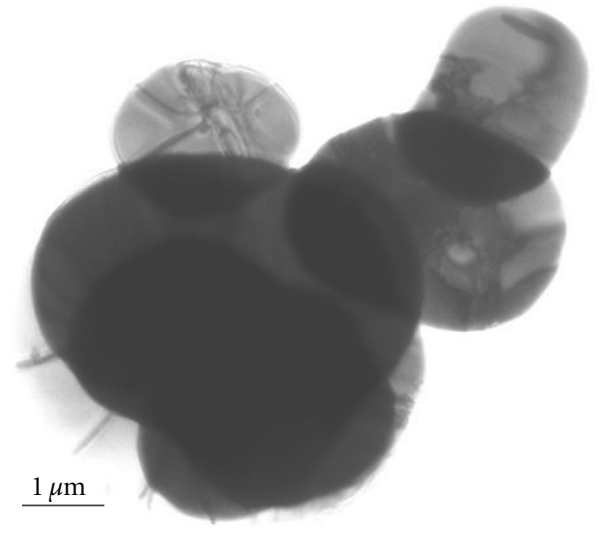

(b) $\beta$ - $\mathrm{BiOBr}$

Figure 2: TEM images of BiOBr.

\section{Results and Discussion}

\subsection{Characterization of Catalyst}

3.1.1. XRD Analysis. Figure 1 shows the XRD patterns of $\mathrm{BiOBr}$. It showed that the diffraction peaks of $\alpha-\mathrm{BiOBr}$ and $\beta$-BiOBr accorded well with the diffraction peak of $\mathrm{P}_{4} / \mathrm{nmm} \mathrm{BiOBr}$ (JCPDS 073-2061). No impurity peak in the XRD patterns indicates the high purity of the samples. Furthermore, the intense peak intensity of two products illustrates the high crystallinity of products and the as-prepared $\alpha$-BiOBr was better crystallized than $\beta$-BiOBr.

3.1.2. TEM Analysis. The TEM images of the products are shown in Figure 2. From Figure 2(a), $\alpha$-BiOBr is layered nanosheet with uniform morphology and smooth surface and, the width of $\alpha-\mathrm{BiOBr}$ is approximately $150 \mathrm{~nm}$. Figure 2(b) shows that $\beta-\mathrm{BiOBr}$ is also neat and smooth layered nanopiece. The size of $\beta$ - $\mathrm{BiOBr}$ is approximately $400 \mathrm{~nm}$, bigger than $\alpha$-BiOBr. This may attribute to that the surfactant $\mathrm{CPB}$ can form a reactor with specific size and morphology in the preparation process, which can control the particle size and morphology of the products in some extent.

3.1.3. BET Analysis. The surface area is an important key on the photocatalytic activities of the photocatalysts generally, the smaller size of the photocatalyst, the larger specific surface area, and the more active sites on granular surface, thus the activity of catalyst can be improved. Figure 3 shows the nitrogen adsorption-desorption isothermal curves of $\mathrm{BiOBr}$ prepared by two methods. The curves of two products in low pressure region are quite gentle. The adsorption increased slowly with the increase of $P / P_{0}$ indicating $\mathrm{N}_{2}$ monolayer adsorption on hole wall in this stage. When $P / P_{0}$ was $0.9-1.0$, the adsorption sharply increased and produced sudden jump, which was caused by the capillary condensing phenomenon of $\mathrm{N}_{2}$ in the mesoporous. The slope of $\mathrm{N}_{2}$ adsorption curve in this stage could be served to measure the homogeneity of mesoporous, and the greater the absolute value of the curve slope, the narrower the pore distribution [21]. Both the slopes of curves (a) and (b) were very great, accounting for that the pore diameter distributions of them 


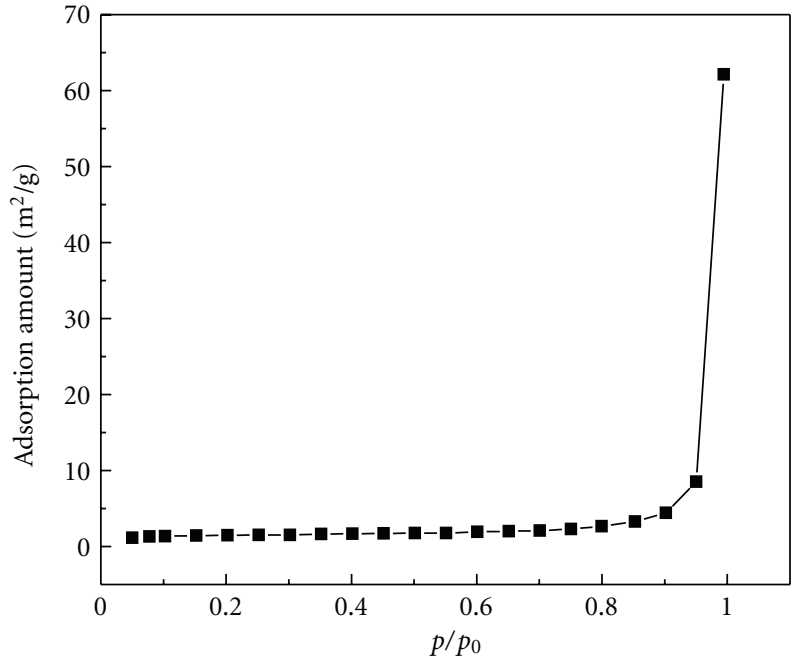

(a) $\alpha-\mathrm{BiOBr}$

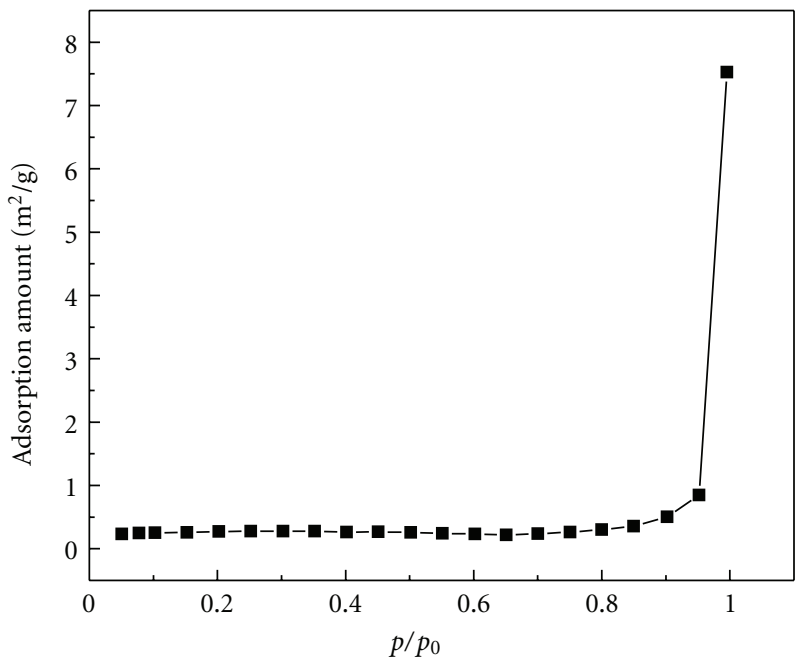

(b) $\beta-\mathrm{BiOBr}$

FIgURE 3: $\mathrm{N}_{2}$ adsorption-desorption isotherms of $\mathrm{BiOBr}$.

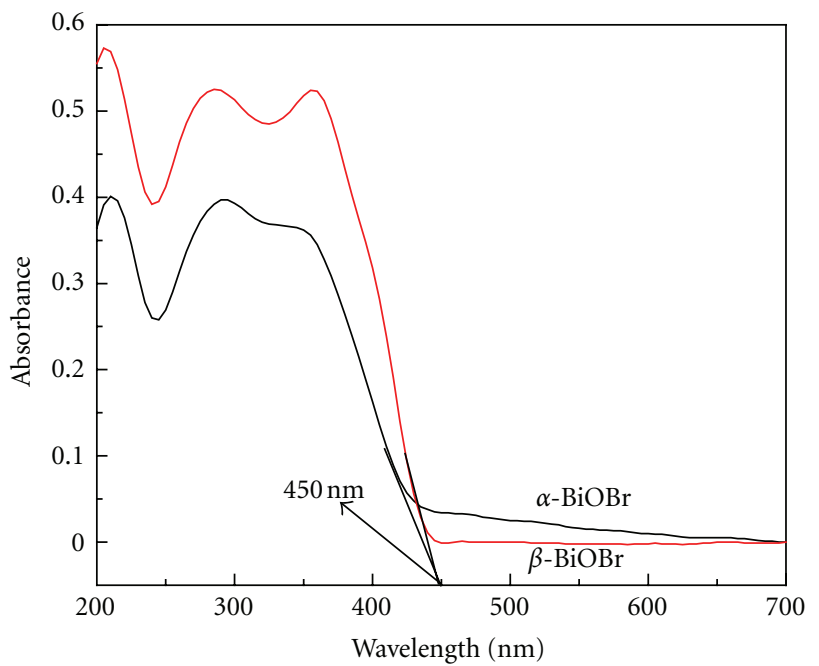

FIgURE 4: UV-Vis diffuse reflectance spectral of $\alpha$-BiOBr and $\beta$ $\mathrm{BiOBr}$.

were narrow. The curves in two pictures were similar which indicated that the morphology, pore size, and surface area of two products were similar, but the specific surface area of $\alpha-\mathrm{BiOBr}$ and $\beta-\mathrm{BiOBr}$ are different, which were about $4.706 \mathrm{~m}^{2} / \mathrm{g}$ and $0.8524 \mathrm{~m}^{2} / \mathrm{g}$, respectively. So $\alpha$-BiOBr had a larger specific surface area, which may be beneficial to reduce the electron-hole recombination.

3.1.4. UV-Vis Diffuse Reflectance Spectral Analysis. The UVVis diffuse reflectance spectral of $\alpha-\mathrm{BiOBr}$ and $\beta-\mathrm{BiOBr}$ in the range of 200-700 nm was shown in Figure 4. The absorption peaks of products are steep and had strong absorption, which indicates that the absorption band of $\mathrm{BiOBr}$ is not attributed to the transition from impurity band to the conduction band but directly internally converted from the valence band to the conduction band [22]. Both The absorption threshold of $\alpha-\mathrm{BiOBr}$ and $\beta$-BiOBr was $450 \mathrm{~nm}$, and the band gap of them was $2.76 \mathrm{eV}$ calculated by the formula of $\lambda g=1239.18 / \mathrm{Eg}$.

\subsection{Photodegradation of $S R B$ by $\mathrm{BiOBr}$}

3.2.1. Comparison of Degrading SRB with BiOBr Prepared by Different Methods. Under the identical conditions, SRB was degraded by $\alpha$-BiOBr and $\beta$ - $\mathrm{BiOBr}$ (Figure 5). SRB cannot be degraded by the two catalysts in absence of light (curve $\mathrm{b}$ and $\mathrm{c}$ ) or under visible light without catalysts (curve a). However, SRB can be degraded in presence of the two catalysts under visible light, and the degradation efficiency of $\alpha$-BiOBr was far better than that of $\beta$-BiOBr. The reaction kinetic constants of photodegradation of SRB by $\alpha-\mathrm{BiOBr}$ and $\beta$-BiOBr were $0.00602 \mathrm{~min}^{-1}$ (curve e) and $0.0047 \mathrm{~min}^{-1}$ (curve d), respectively.

3.2.2. The Optimization of Degrading SRB. Photocatalytic reactions mainly occur on the surface of the catalyst, therefore the intrinsic characteristic of the surface determines the photocatalytic activity. But reactions also can be significantly affected by surroundings. The influences of catalyst dosage and $\mathrm{pH}$ on the degradation of SRB by $\alpha$-BiOBr were discussed. The solution $\mathrm{pH}$ has great effect on the surface charge of catalyst, pollutants molecules charge, adsorption of contaminants on catalyst surface, hydroxyl concentration, and so on, which affect the degradation efficiency. Figure 6 showed the degradation of SRB under different pHs. $\alpha$-BiOBr had the best catalytic activity when $\mathrm{pH}$ was about 2 . With the increase of $\mathrm{pH}$, the degradation rates reduced gradually, but the activity reduced when $\mathrm{pH}$ was less than 2 . In addition, catalyst in the neutral and alkaline solution hardly degraded SRB. This showed at the catalytic activity of BiOBr in acidic environment was far better than that in the neutral and alkaline conditions. The probable reason was that the isoelectric point of the $\mathrm{BiOBr}$ is in the acidic range and its 


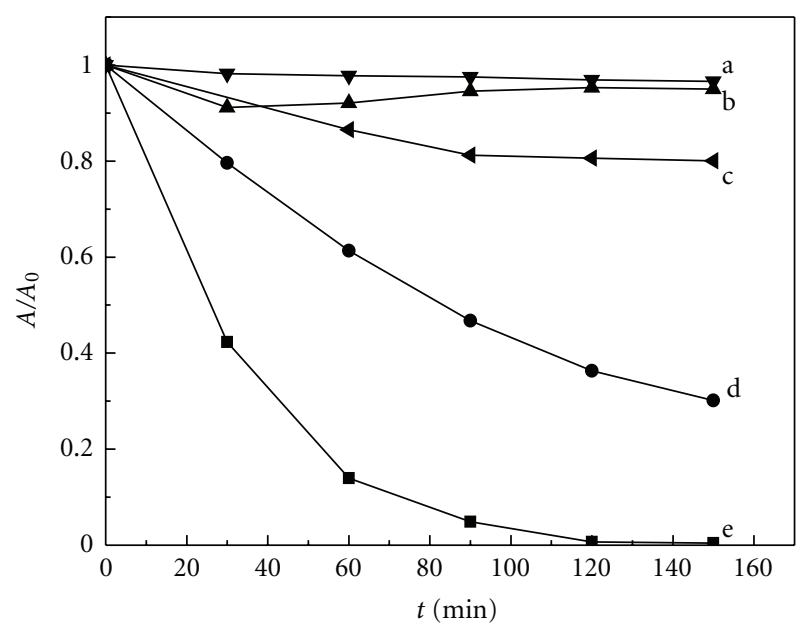

FIGURE 5: Photocatalytic degradation of SRB in different systems, $(\mathrm{SRB})=1.5 \times 10^{-5} \mathrm{~mol} / \mathrm{L},(\mathrm{BiOBr})=0.2 \mathrm{~g} / \mathrm{L}, \mathrm{pH}=3$, (a) SRB $/ \mathrm{Vis}$; (b) $\beta-\mathrm{BiOBr} / \mathrm{SRB} / \mathrm{Dark}$; (c) $\alpha-\mathrm{BiOBr} / \mathrm{SRB} / \mathrm{Dark}$; (d) $\beta$-BiOBr $/ \mathrm{SRB} /$ Vis; (e) $\alpha$-BiOBr/SRB/Vis.

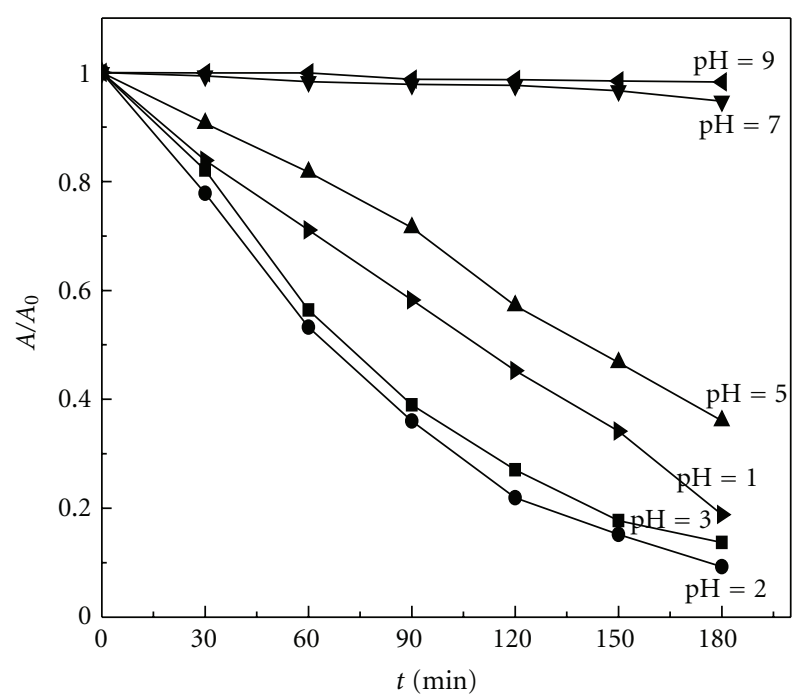

Figure 6: Effect of $\mathrm{pH},(\mathrm{SRB})=1.5 \times 10^{-5} \mathrm{~mol} / \mathrm{L},(\mathrm{BiOBr})=0.2 \mathrm{~g} / \mathrm{L}$.

surface is negatively charged. BiOBr cannot combine dye easily when $\mathrm{pH}$ is larger, so the degradation rates were very small in basic and neutral solution. Figure 7 showed the influence of catalyst dosage on the catalytic efficiency. The degradation rates increased with the catalytic concentration. When the dosage was $0.8 \mathrm{~g} / \mathrm{L}, \mathrm{BiOBr}$ had the best catalytic efficiency. If the amount of catalyst continued to increase, the degradation rate unchanged and even decreased. It may be attributed to active centers provided by $\mathrm{BiOBr}$ increase with the amount of catalyst, so $\mathrm{BiOBr}$ can absorb more photons and pollutants to efficiently degrade SRB. However, when the catalyst dosage is too much, the scattering effect enhances and the efficient absorption of light reduces, so the degradation efficiency declines [23].

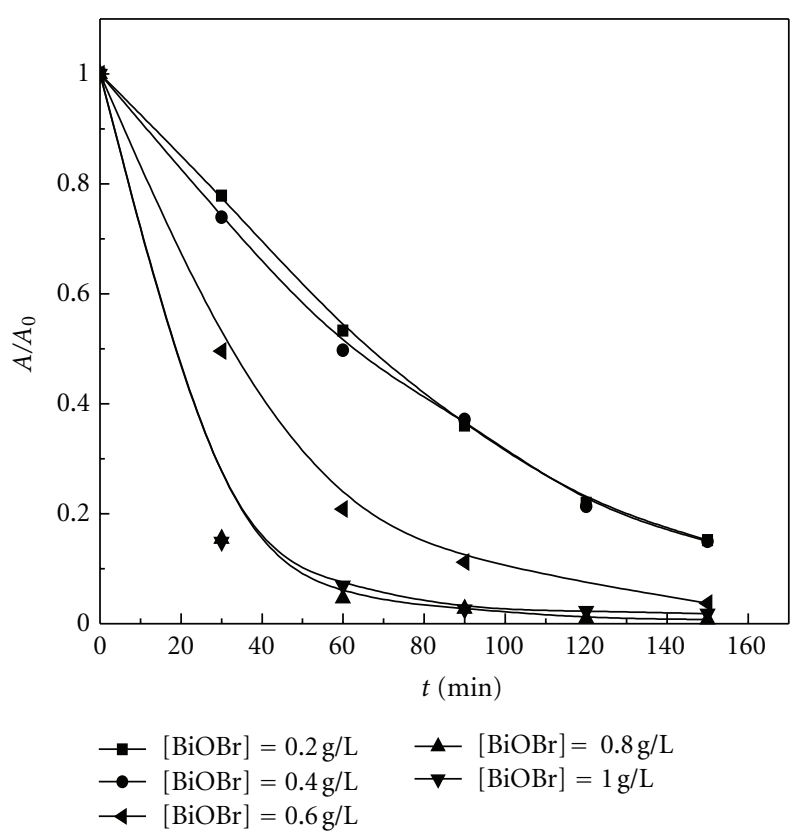

Figure 7: Effect of catalyst dosage, $(\mathrm{SRB})=1.5 \times 10^{-5} \mathrm{~mol} / \mathrm{L}, \mathrm{pH}=$ 2.

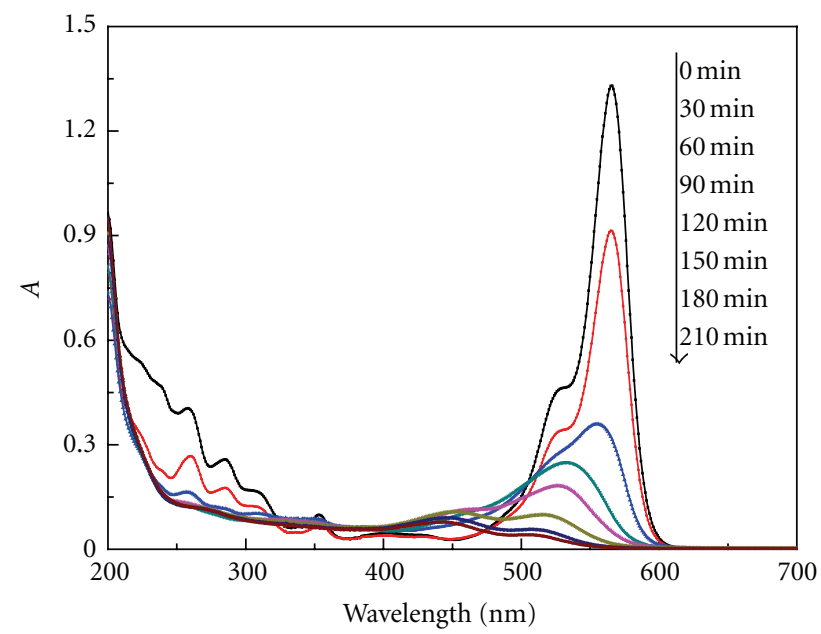

FIgURE 8: Temporal UV-Vis spectra changes of SRB, $(\mathrm{SRB})=1.5 \times$ $10^{-5} \mathrm{~mol} / \mathrm{L},(\mathrm{BiOBr})=0.8 \mathrm{~g} / \mathrm{L}, \mathrm{pH}=2$.

3.2.3. Degradation of SRB under the Optimum Conditions. The degradation of SRB under the optimum conditions $(\mathrm{pH}=2,[\mathrm{BiOBr}]=0.8 \mathrm{~g} / \mathrm{L})$ was presented in Figure 8. The characteristic absorption peak $(\lambda=565 \mathrm{~nm})$ of SRB decreased constantly and showed a blue shift, which demonstrated that the conjugated structure of SRB decreased and the molecule suffered destruction. After $150 \mathrm{~min}$, the absorption of SRB in visible light disappeared completely and the decoloration rate reached $100 \%$.

3.2.4. Cyclic Photocatalytic Degradation of SRB. The stability and reusability of semiconductors are important parameters to measure their practical value. In order to discuss the 


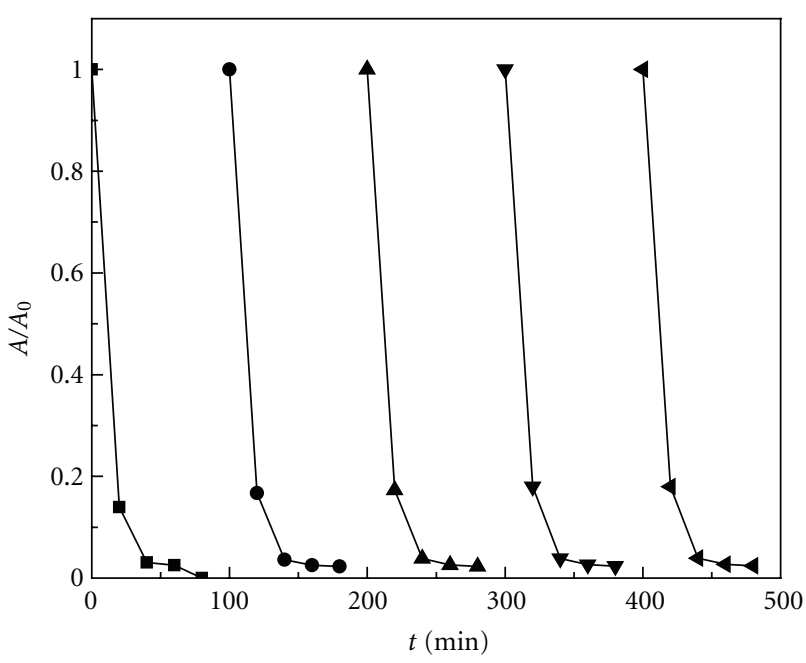

FIgURE 9: The degradation curve of SRB during multicycle degradation process, $(\mathrm{SRB})=1.5 \times 10^{-5} \mathrm{~mol} / \mathrm{L},(\mathrm{BiOBr})=0.8 \mathrm{~g} / \mathrm{L}, \mathrm{pH}=$ 2.

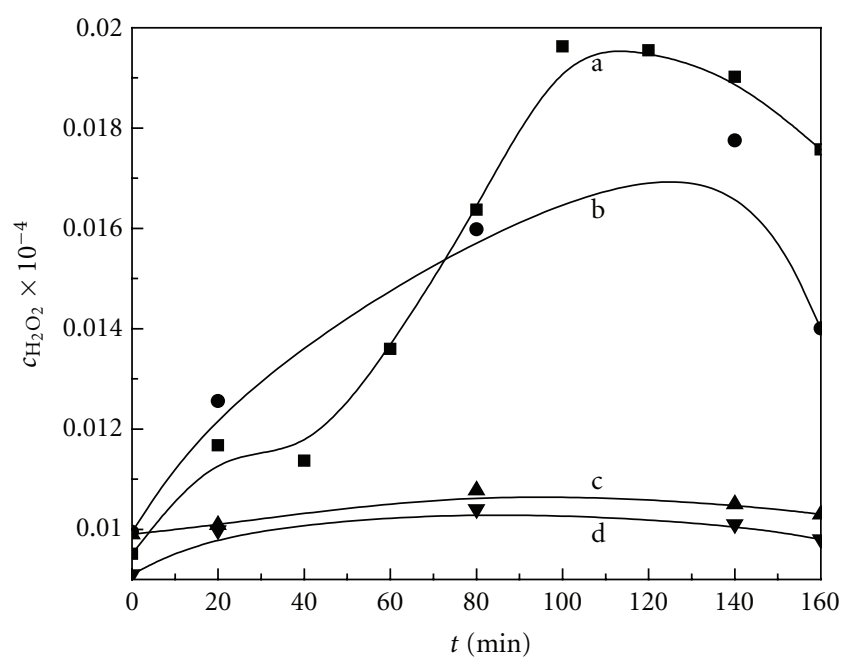

Figure 10: Changes of $\mathrm{H}_{2} \mathrm{O}_{2}$ during the degradation process of SRB, (a) $\beta$-BiOBr/SRB/Vis; (b) $\alpha$-BiOBr/SRB/Vis; (c) $\alpha$ $\mathrm{BiOBr} / \mathrm{SRB} / \mathrm{Dark}$; (d) $\beta$-BiOBr$/ \mathrm{SRB} / \mathrm{Vis},(\mathrm{SRB})=1.5 \times 10^{-5} \mathrm{~mol} / \mathrm{L}$, $(\mathrm{BiOBr})=0.8 \mathrm{~g} / \mathrm{L}, \mathrm{pH}=2$.

stability and repeatable usability of $\mathrm{BiOBr}, \alpha$ - $\mathrm{BiOBr}$ was used to circularly degrade SRB under the optimum conditions (Figure 9). After recycling for 5 times, the degradation efficiency of SRB did not reduce obviously. BiOBr could completely degrade SRB after 100 min, which indicated $\mathrm{BiOBr}$ has good stability, reusability, and application potential.

\subsection{The Measurement of Intermediate Products during the Degradation of $S R B$}

3.3.1. The Measurement of $\mathrm{H}_{2} \mathrm{O}_{2}$. The change of $\mathrm{H}_{2} \mathrm{O}_{2}$ in the degradation of SRB by $\alpha-\mathrm{BiOBr}$ and $\beta-\mathrm{BiOBr}$ was measured by $\mathrm{DPD}$ method. As shown in Figure 10, $\mathrm{H}_{2} \mathrm{O}_{2}$ did not generate in the dark in presence of $\alpha$ - $\mathrm{BiOBr}$ and $\beta$ - $\mathrm{BiOBr}$ (curve $c$ and $d$ ). Under visible light irradiation, $\mathrm{BiOBr}$ was stimulated to produce electrons and holes, which reacted with dissolved oxygen and water adsorbed on the surface of catalysts to produce $\mathrm{H}_{2} \mathrm{O}_{2}$. At the beginning of reaction, $\mathrm{H}_{2} \mathrm{O}_{2}$ was accumulated and then gradually consumed to resolve into ${ }^{\bullet} \mathrm{OH}$ which had better oxidizability to degrade $\mathrm{SRB}$. During the accumulation stage, the generation rate of $\mathrm{H}_{2} \mathrm{O}_{2}$ in $\alpha$ - $\mathrm{BiOBr} / \mathrm{SRB}$ system (curve b) is larger than that in $\beta$-BiOBr/SRB system (curve a). The degradation rate of SRB in the former system is larger than that in the latter system, so $\mathrm{H}_{2} \mathrm{O}_{2}$ is consumed more rapidly and the total amount of $\mathrm{H}_{2} \mathrm{O}_{2}$ is less.

3.3.2. The Measurement of Active Species. The oxidation species involved in photocatalytic oxidation reaction are mainly ${ }^{\bullet} \mathrm{OH}$ and $\mathrm{O}_{2}{ }^{\bullet-}$, which have high oxidation potential and can oxidize almost all organic compounds. The ESR technology is a very effective method to measure the short life of the radicals. Figure 11 showed the characteristic signal peak of DMPO- ${ }^{\bullet} \mathrm{OH}$ and $\mathrm{DMPO}-\mathrm{O}_{2}{ }^{-{ }^{-}}$in different systems. The characteristic quartet peaks of DMPO- ${ }^{\circ} \mathrm{OH}$ adducts with an intensity ratio of $1: 2: 2: 1$ appeared, which showed that ${ }^{\bullet} \mathrm{OH}$ is generated in the catalytic oxidation process of $\mathrm{SRB}$ by $\mathrm{BiOBr}$, and that the catalytic mechanism mainly involved the ${ }^{\bullet} \mathrm{OH}$ oxidation mechanism. The ESR signals of DMPO- $\mathrm{O}_{2}{ }^{--}$adducts were also observed in the system under visible radiation (Figure 11), but the intensity of the signal was faint, which indicated that $\mathrm{O}_{2}{ }^{--}$was also produced under visible light, but the main oxidation species was ${ }^{\bullet} \mathrm{OH}$.

3.3.3. The Deep Mineralization of SRB. Figure 12 showed the changes of total organic carbon (TOC) in the degradation process of SRB. From the results, the TOC value gradually reduced. Under the same reaction conditions, the TOC removal rates of SRB degraded with $\alpha-\mathrm{BiOBr}$ and $\beta-\mathrm{BiOBr}$ are $86 \%$ and $48 \%$, respectively, after $900 \mathrm{~min}$, which demonstrated that $\mathrm{BiOBr}$ prepared with $\mathrm{CPB}$ has a better mineralization of pollutants.

3.3.4. IR Analysis. Figure 13 presented the IR spectrum of SRB degraded by $\alpha$-BiOBr in different photocatalytic reaction time. It is known that the bands at 1460, 1500, 1580, and $1600 \mathrm{~cm}^{-1}$ are corresponded to the stretching vibration of benzenes in SRB molecules and the band at $1649 \mathrm{~cm}^{-1}$ is due to the vibration of C-N stretching. The band at 1460 $1508 \mathrm{~cm}^{-1}$ is caused by the vibration of the heterocycles in dye molecules. The band at $1330 \mathrm{~cm}^{-1}$ is due to the vibration of C-Ar peak, and the bands at $1120 \mathrm{~cm}^{-1}$ and 665 $620 \mathrm{~cm}^{-1}$ are attributed to the vibration of the $-\mathrm{SO}_{3}{ }^{-}$functional groups. With the increase of reaction time, some characteristic peaks of $\mathrm{SRB}$ disappeared, and new peaks at $1650 \mathrm{~cm}^{-1}$ and $1400 \mathrm{~cm}^{-1}$, attributed to the vibration of $\mathrm{C}=\mathrm{O}$ expansive and diethylamine- $\mathrm{CH}_{3}$, appeared gradually. The results showed that the conjugate structure of SRB was damaged during the process of catalytic degradation and further demonstrated that SRB was not only simply discolored, but also deeply degrade.

3.3.5. CV Analysis. Cyclic voltammetry provides a wealth of information in researching electrode process via analyzing 

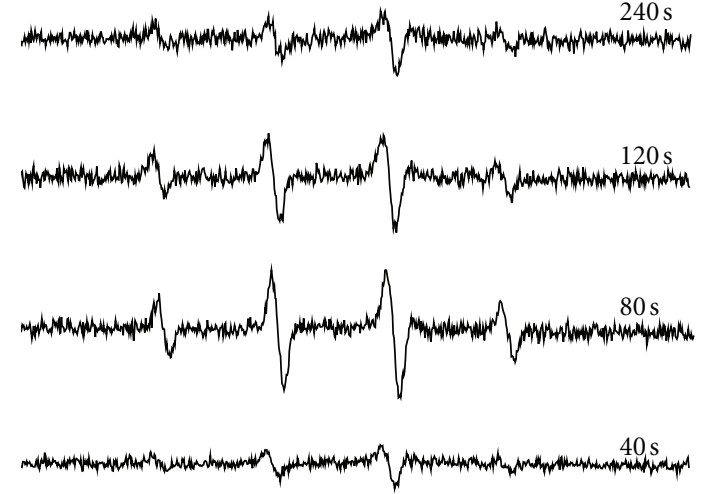

Magnetic field (G)

(a)
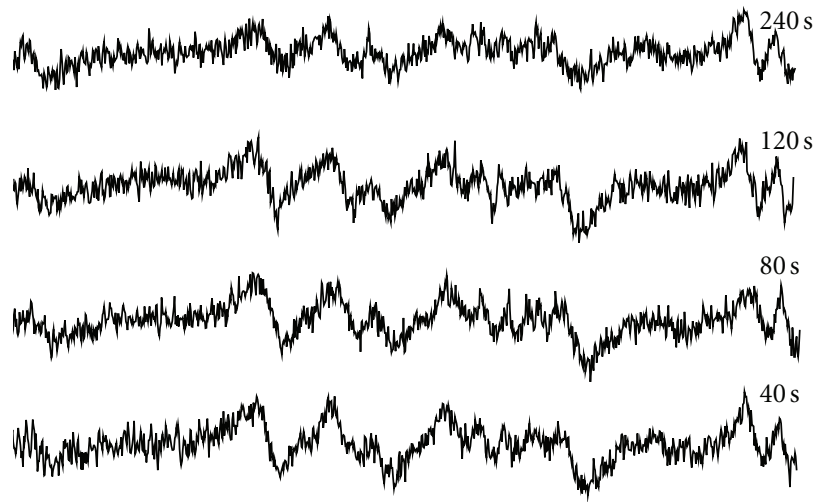

Magnetic field (G)

(b)

FIgURE 11: ESR signals of the DMPO- ${ }^{-} \mathrm{OH}$ adducts (a) and $\mathrm{DMPO}-\mathrm{O}_{2}{ }^{--}$adducts (b).

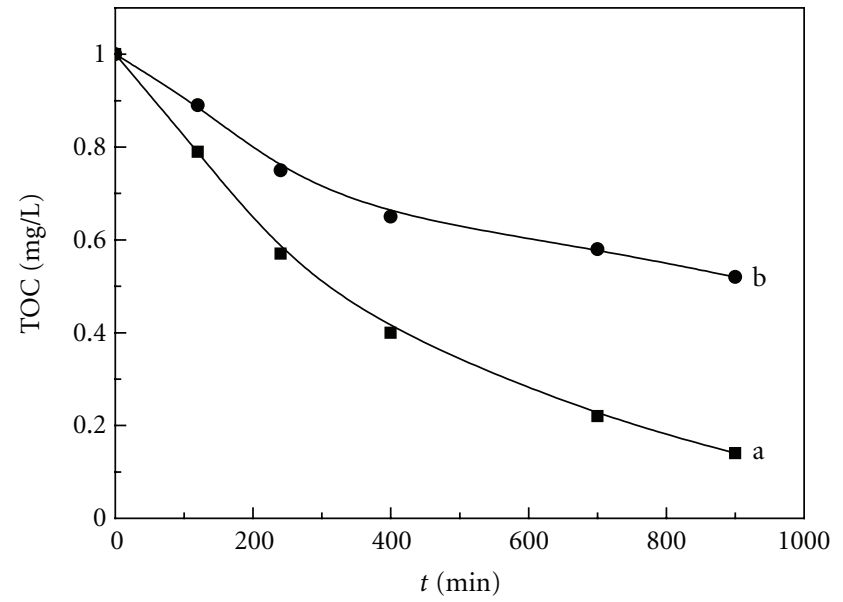

FIgURE 12: TOC removals during the degradation process of $\mathrm{SRB}$, (a) $\alpha-\mathrm{BiOBr} / \mathrm{SRB} / \mathrm{Vis}$; (b) $\beta-\mathrm{BiOBr} / \mathrm{SRB} / \mathrm{Vis},(\mathrm{SRB})=3.0 \times$ $10^{-5} \mathrm{~mol} / \mathrm{L},(\mathrm{BiOBr})=0.8 \mathrm{~g} / \mathrm{L}, \mathrm{pH}=2$.

the shape of the scanning curve [24], which can account for the photocatalytic phenomenon. Figure 14 was the currentvoltage curves of two products under the same condition. $\alpha$ $\mathrm{BiOBr}$ had two pairs of redox peaks. The oxidation potential was $0.126 \mathrm{~V}$ and $0.407 \mathrm{~V}$ and reduction potential was $0.259 \mathrm{~V}$ and $0.441 \mathrm{~V}$. While $\beta$-BiOBr only had a pair of redox peak. The oxidation and reduction peaks were $0.259 \mathrm{~V}$ and $0.436 \mathrm{~V}$, respectively. This showed that there are two different electronic transfer between the two different valence states of $\alpha-\mathrm{BiOBr}$ which may react two redox reactions; while $\beta$ $\mathrm{BiOBr}$ only react one redox reaction. And it also showed that the transformation of electrons on $\alpha$ - $\mathrm{BiOBr}$ surface is more easy than that on $\beta$ - $\mathrm{BiOBr}$, thus the photocatalytic activity of $\alpha-\mathrm{BiOBr}$ is higher than $\beta$ - $\mathrm{BiOBr}$.

3.4. Photocatalytic Degradation of Salicylic Acid. Salicylic acid (SA) is a kind of small colorless molecule which has

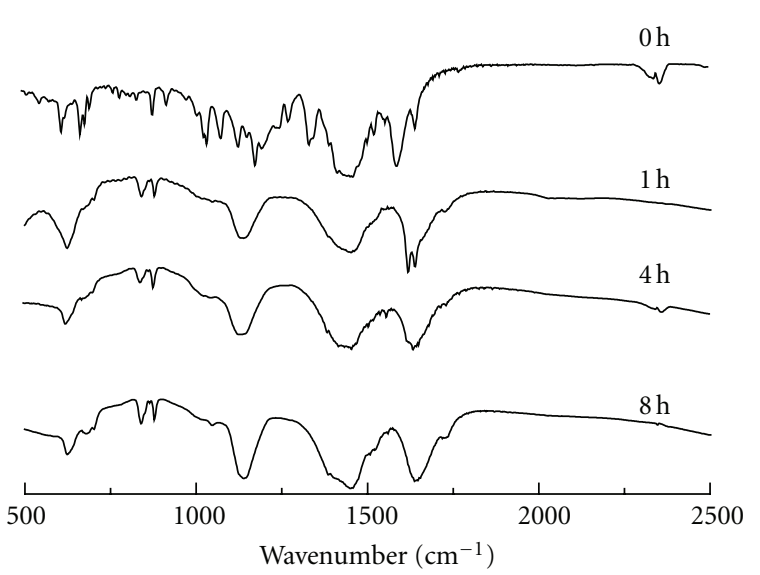

FIGURE 13: IR analysis of degrading SRB, $(\mathrm{SRB})=3.0 \times 10^{-5} \mathrm{~mol} / \mathrm{L}$, $(\mathrm{BiOBr})=0.8 \mathrm{~g} / \mathrm{L}, \mathrm{pH}=2$.

no absorption in visible light, so it is usually used to characterize the visible photoreactivity of photocatalysts. The degradation of SA was under the illumination of visible light $(\lambda \geq 420 \mathrm{~nm})$ and the concentration of it was measured by HPLC (Figure 15). Curve a showed that SA was not photodissociated under visible irradiation. Curve $\mathrm{b}$ and $\mathrm{c}$ showed that $\alpha$ - $\mathrm{BiOBr}$ and $\beta$-BiOBr can adsorb little amount of SA. Curve $\mathrm{d}$ and e represented the degradation of SA by $\alpha-\mathrm{BiOBr}$ and $\beta$-BiOBr under visible light. After $8 \mathrm{~h}$, the degradation rates of SA by $\alpha$ - $\mathrm{BiOBr}$ and $\beta$-BiOBr were $97 \%$ and $37.3 \%$, respectively. The results further indicated that $\alpha$ $\mathrm{BiOBr}$ had a better catalytic activity than $\beta-\mathrm{BiOBr}$ and could be stimulated effectively by visible light to degrade small molecular pollutants.

\section{Conclusions}

In summary, we synthesized layered $\mathrm{BiOBr}$ with high catalytic activity by using different reactants. CPB not only 


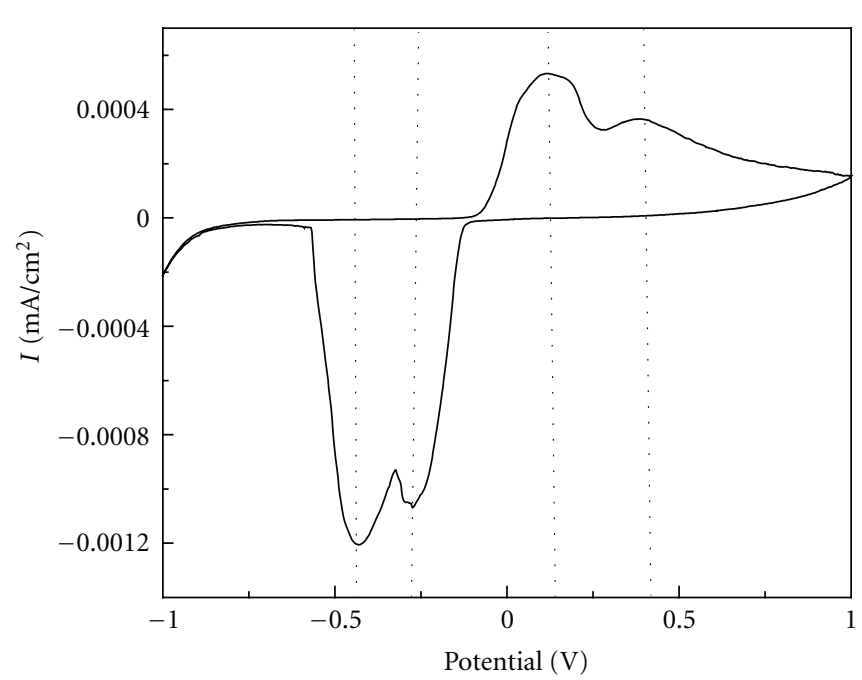

(a) $\alpha-\mathrm{BiOBr}$

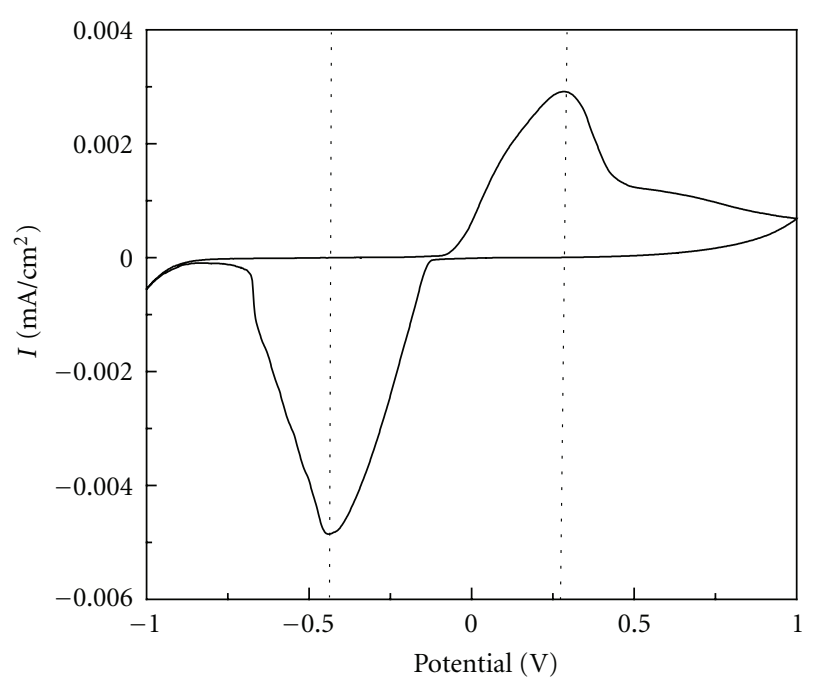

(b) $\beta-\mathrm{BiOBr}$

Figure 14: The cyclic voltammetry of BiOBr.

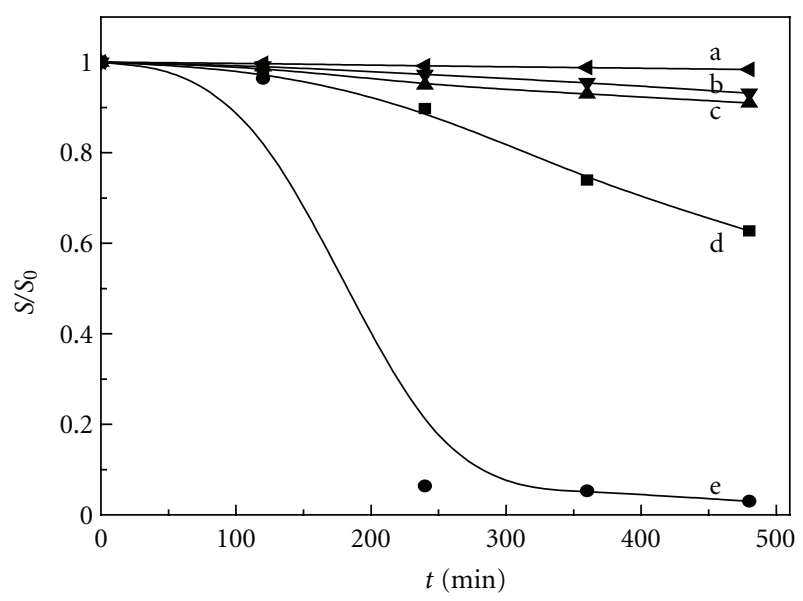

Figure 15: Photodegradation of SA, (a) SA/Vis; (b) $\beta$ $\mathrm{BiOBr} / \mathrm{SA} / \mathrm{Dark} ; \quad$ (c) $\alpha$-BiOBr/SA/Dark; (d) $\beta$-BiOBr/SA/Vis; (e) $\alpha-\mathrm{BiOBr} / \mathrm{SA} / \mathrm{Vis},(\mathrm{SA})=5 \mathrm{~m} \mathrm{~mol} / \mathrm{L},(\mathrm{BiOBr})=0.8 \mathrm{~g} / \mathrm{L}$.

can be used as bromine sources to prepare $\mathrm{BiOBr}$, but also is served as template to form a reactor with specific size and morphology in the preparation process, which can control the particle size and morphology of the products in some extent. Obviously, $\mathrm{BiOBr}$ synthesized by $\mathrm{CPB}$ had a better efficiency on degrading SRB and SA under visible irradiation than $\mathrm{BiOBr}$ prepared by $\mathrm{NaBr}$ which only served as bromine sources. The different products showed different particle size, surface area, and adsorption quantity, which result in different degradation efficiency on pollutants.

\section{Acknowledgments}

This work was funded by the National Natural Science Foundation of China (nos. 21177072, 20877048), Innovation group project of Hubei Province Natural Science Foundation (no. 2009CDA020), and Open Foundation about Key Laboratory of Catalysis and Materials Science of the State Ethnic Affairs Commission \& ministry of Education of Hubei Province (no. CHCL10007).

\section{References}

[1] J. E. D. Davies, "Solid state vibrational spectroscopy-III[1] The infrared and raman spectra of the bismuth(III) oxide halides," Journal of Inorganic and Nuclear Chemistry, vol. 35, no. 5, pp. 1531-1534, 1973.

[2] H. Deng, J. Wang, Q. Peng, X. Wang, and Y. Li, "Controlled hydrothermal synthesis of bismuth oxyhalide nanobelts and nanotubes," Chemistry, vol. 11, no. 22, pp. 6519-6524, 2005.

[3] Z. Ai, W. Ho, and S. Lee, "Efficient visible light photocatalytic removal of NO with BiOBr-graphene nanocomposites," Journal of Physical Chemistry C, vol. 115, no. 51, pp. 25330-25337, 2011.

[4] D. Zhang, M. Wen, B. Jiang, G. Li, and J. C. Yu, "Ionothermal synthesis of hierarchical $\mathrm{BiOBr}$ microspheres for water treatment," Journal of Hazardous Materials, vol. 211-212, pp. 104$111,2012$.

[5] C. Yu, W. Zhou, J. Yu, F. Cao, and X. Li, “Thermal stability, microstructure and photocatalytic activity of the bismuth oxybromide photocatalyst," Chinese Journal of Chemistry, vol. 30, no. 3, pp. 721-726, 2012.

[6] H. J. Li and W. P. He, "The dispersibility and catalytic activity researches of Nano-TiO ${ }_{2}$," Paint and Coatings Industry, vol. 1, no. 40, pp. 24-28, 2010.

[7] H. W. Ji, W. H. Ma, Y. P. Huang, J. C. Zhao, and Z. P. Wang, "Study on visible light abduction of $\mathrm{TiO}_{2}$ of photocatalyst," Chinese Science Bulletin, vol. 48, no. 21, pp. 2199-2204, 2003.

[8] Q. Liu, "The applications and its prospects of $\mathrm{TiO}_{2}$ in wastewater treatment," Environmental Sciences, vol. 2, pp. 122123, 2010.

[9] W. L. Huang and Q. Zhu, "Electronic structures of relaxed BiOX (X = F, Cl, Br, I) photocatalysts," Computational Materials Science, vol. 43, no. 4, pp. 1101-1108, 2008. 
[10] K. L. Zhang, C. M. Liu, F. Q. Huang, C. Zheng, and W. D. Wang, "Study of the electronic structure and photocatalytic activity of the BiOCl photocatalyst," Applied Catalysis B, vol. 68, no. 3-4, pp. 125-129, 2006.

[11] N. Kijima, K. Matano, M. Saito et al., "Oxidative catalytic cracking of n-butane to lower alkenes over layered $\mathrm{BiOCl}$ catalyst," Applied Catalysis A, vol. 206, no. 2, pp. 237-244, 2001.

[12] J. Henle, P. Simon, A. Frenzel, S. Scholz, and S. Kaskel, "Nanosized $\mathrm{BiOX}(\mathrm{X}=\mathrm{Cl}, \mathrm{Br}, \mathrm{I})$ particles synthesized in reverse microemulsions," Chemistry of Materials, vol. 19, no. 3, pp. 366-373, 2007.

[13] Z. Deng, D. Chen, B. Peng, and F. Tang, "From bulk metal Bi to two-dimensional well-crystallized $\mathrm{BiOX}(\mathrm{X}=\mathrm{Cl}, \mathrm{Br})$ microand nanostructures: synthesis and characterization," Crystal Growth and Design, vol. 8, no. 8, pp. 2995-3003, 2008.

[14] Y. Huo, J. Zhang, M. Miao, and Y. Jin, "Solvothermal synthesis of flower-like $\mathrm{BiOBr}$ microspheres with highly visible-light photocatalytic performances," Applied Catalysis B, vol. 111112, pp. 334-341, 2012.

[15] X. Zhang, Z. Ai, F. Jia, and L. Zhang, "Generalized one-pot synthesis, characterization, and photocatalytic activity of hierarchical BiOX (X = Cl, Br, I) nanoplate microspheres," Journal of Physical Chemistry C, vol. 112, no. 3, pp. 747-753, 2008.

[16] M. C. Su and Y. J. Wang, "LaOBr-BiOCl and its photism," Higher Chemistry Journal, vol. 5, pp. 12-15, 1982.

[17] M. Shang, W. Wang, and L. Zhang, "Preparation of BiOBr lamellar structure with high photocatalytic activity by CTAB as Br source and template," Journal of Hazardous Materials, vol. 167, no. 1-3, pp. 803-809, 2009.

[18] L. Zhang, X. F. Cao, X. T. Chen, and Z. L. Xue, "BiOBr hierarchical microspheres: microwave-assisted solvothermal synthesis, strong adsorption and excellent photocatalytic properties," Journal of Colloid and Interface Science, vol. 354, no. 2, pp. 630-636, 2011.

[19] Y. F. Fang, Y. P. Huang, D. F. Liu, Y. Huang, W. Guo, and J. David, "The comparative study of nitrogen mixed $\mathrm{TiO}_{2}$ degrading organic pollutants in visible light," Environmental Science, vol. 19, pp. 97-102, 2007.

[20] F. A. Villamena and J. L. Zweier, "Detection of reactive oxygen and nitrogen species EPR spin trapping," Antioxidants and Redox Signaling, vol. 6, no. 3, pp. 619-629, 2004.

[21] H. F. Zhou, Y. J. Yang, and J. S. Zhang, "The synthesis and catalytic performance of miscellaneous atomic MCM-41 molecular sieve," Journal of Materials Research, vol. 22, no. 2, pp. 199-204, 2009.

[22] J. Zhang, F. Shi, J. Lin et al., "Self-assembled 3-D architectures of $\mathrm{BiOBr}$ as a visible light-driven photocatalyst," Chemistry of Materials, vol. 20, no. 9, pp. 2937-2941, 2008.

[23] N. S. Deng and F. Wu, Environment Photochemical, Chemical Industry Press, Beijing, China, 2003.

[24] X. L. Liu, The influence of Cerium on $\mathrm{TiO}_{2}$ photocatalytic activity [M.S. thesis], Beijing Chemical University, 2003. 

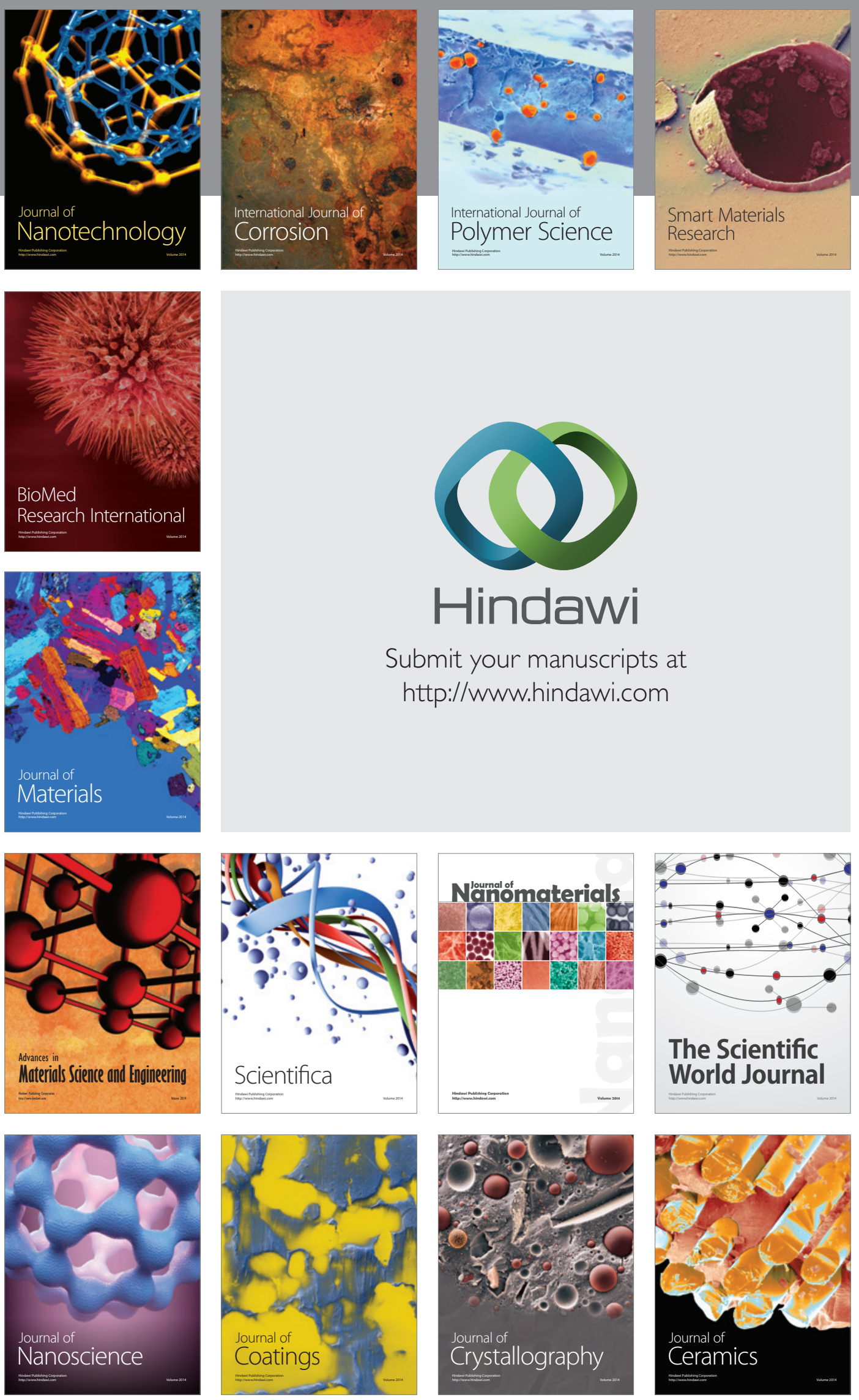

The Scientific World Journal

Submit your manuscripts at

http://www.hindawi.com

\section{World Journal}

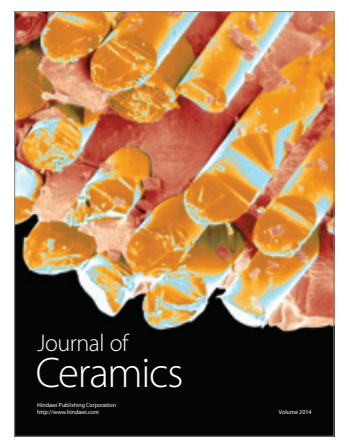

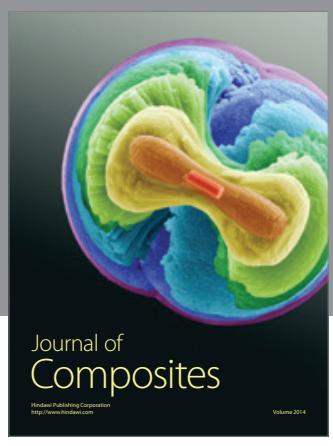
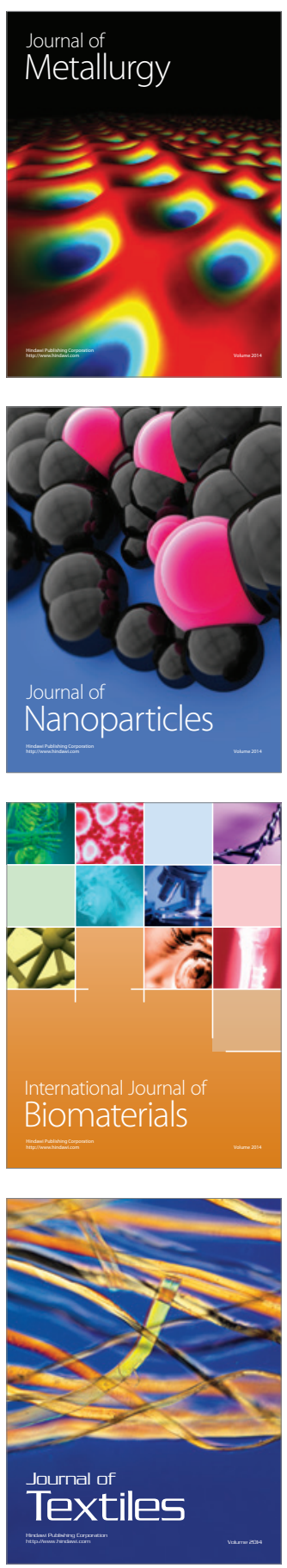